\title{
Combining series elastic actuation and magneto-rheological damping for the control of agile locomotion
}

\author{
E. Garcia *, J.C. Arevalo, G. Muñoz, P. Gonzalez-de-Santos \\ Robot Locomotion and Interaction Group, Centre for Automation and Robotics-CSIC-UPM, 28500 Madrid, Spain
}

\begin{abstract}
A B S T R A C T
All-terrain robot locomotion is an active topic of research. Search and rescue maneuvers and exploratory missions could benefit from robots with the abilities of real animals. However, technological barriers exist to ultimately achieving the actuation system, which is able to meet the exigent requirements of these robots. This paper describes the locomotion control of a leg prototype, designed and developed to make a quadruped walk dynamically while exhibiting compliant interaction with the environment. The actuation system of the leg is based on the hybrid use of series elasticity and magneto-rheological dampers, which provide variable compliance for natural-looking motion and improved interaction with the ground. The locomotion control architecture has been proposed to exploit natural leg dynamics in order to improve energy efficiency. Results show that the controller achieves a significant reduction in energy consumption during the leg swing phase thanks to the exploitation of inherent leg dynamics. Added to this, experiments with the real leg prototype show that the combined use of series elasticity and magneto-rheological damping at the knee provide a $20 \%$ reduction in the energy wasted in braking the knee during its extension in the leg stance phase.
\end{abstract}

Agile legged robots

Large power-to-weight actuators

Hybrid actuators for robotics

Gait control

Compliance control

\section{Introduction}

Legged robots envisaged for field applications in natural, unstructured environments, should have the locomotive abilities of real animals. Considering that one half of the world's landmass is not accessible to current wheeled and tracked vehicles [1], but that animals using their legs can go almost anywhere, legged vehicles, emulating their biological counterparts, are the best choice for field missions in a natural environment. However, it is well known that most of the current legged locomotion devices feature high complexity and low speed, particularly if high payloads have to be transported (see Table 1). Although in the past 40 years several research groups have been involved in achieving artificial legged locomotion (a comprehensive survey can be found in [2]), the problem of power presented by legged robots is still unsolved and requires a strong research effort.

We use the term "agile legged robot" to refer to a class of ground vehicle, propelled by robotic legs, which feature similar characteristics as what US DARPA (Defense Advanced Research projects Agency) terms "agile ground vehicle", a product of the DARPA's Unmanned Ground Combat Vehicle (UGCV) program [3]. The Agile UCV concept incorporates a combination of technologies and design features which provide optimum performance against mobility, payload, and endurance goals. Concretely, an agile ground vehicle in the UGCV program featured a payload-to-weight ratio requirement of 0.7 . Added to this static load requirement, a quadruped robot, to walk dynamically, must make a thrust to the ground yielding dynamic impact loads that could exceed three times the static load on the supporting leg [4]. Therefore, dynamic load requirements exceed static payload requirements in agile legged robots, which has to be considered at the structural design stage. In a trot gait, two legs thrust the ground simultaneously, so the static load on each leg is at least one half of the robot weight. Thus, the legs of an agile robot should be able to support dynamic loads larger than 1.5 times the robot weight.

Regarding robot forward speed, it is widely known that a leg cannot propel a body as fast as a wheel can do. In order to use a measure of speed independent of animal/robot size, we will use the dimensionless speed computed from the Froude number (FR) as $\hat{u}=\sqrt{F R}[5]$, where

$F R=\frac{v^{2}}{g L}$

being $v$, the forward speed, g, $9.81 \mathrm{~ms}^{-2}$ and $L$, the characteristic leg length. Biological quadrupeds walking fast (not running) feature a dimensionless speed between 0.54 to 0.7 . Concretely, horses transition from walk to trot at $\hat{u}=0.59$ [6]. As exceeding this dimensionless speed requires the quadruped to run (trot or gallop) and some complex terrains could impede the use of those 
Table 1

Load capacity for dynamic walking, payload-to-weight ratio and maximum dimensionless forward speed of significant quadruped robots developed in the last 12 years.

\begin{tabular}{|c|c|c|c|c|c|}
\hline Robot & Weight (kg) & Dynamic walking & Payload/Weight & Max, dimensionless speed & Year \\
\hline Aibo [7] & 1.65 & No & 0 & 0.35 & 1999 \\
\hline Scout II [8] & 27 & Yes & 0.02 & 1.17 & 1999 \\
\hline SILO4 [9] & 34 & No & 0.59 & 0.06 & 2002 \\
\hline TITAN XI [10] & 7000 & No & 0.06 & 0.003 & 2002 \\
\hline Tekken II [11] & 4.3 & Yes & 0 & 0.65 & 2003 \\
\hline LittleDog [12] & 2.2 & No & $0^{\mathrm{a}}$ & 0.23 & 2005 \\
\hline Tekken 3 \& 4 [13] & 10 & Yes & 0.2 & 0.65 & 2005 \\
\hline Kolt [14] & 90 & Yes & - & 0.35 & 2005 \\
\hline Rush [15] & 4.3 & Yes & 0 & 0.64 & 2007 \\
\hline BigDog [16] & 109 & Yes & 1,41 & 0.72 & $2008^{b}$ \\
\hline
\end{tabular}

attleDog's complex computing is provided by an off-board processor, it is not a self-contained autonomous quadruped [17].

b Although the first BigDog robot was developed in 2005, this data corresponds to the 2008 BigDog prototype.

high-speed gaits, we have considered the dimensionless speed of 0.54 as the lower speed limit for a legged robot to be considered agile.

Table 1 lists most relevant quadruped robots developed in the last 12 years, showing their leg capability of withstanding dynamic loads of 1.5 times the robot's weight, payload-to-weight ratios and maximum dimensionless forward speed, which has been computed from robot dimensions and speed published by the robot's authors. It is clear that almost none of them reach the marks to be considered an agile legged robot. Although some research labs are working in this direction-Stanford University [18], Carnegie Mellon University [19], Georgia Institute of Technology [20], University of Michigan [21], Italian Institute of Technology [22]-to these authors' best knowledge the only existing quadruped with agile locomotion abilities is BigDog [16], a robot being developed at Boston Dynamics (USA). Unfortunately, technical and scientific details on BigDog project are not available to the research community, probably due to IPR protection.

However, it is not just about power. Achieving agile locomotion abilities on natural terrain requires compliant adaptation to the ground and energy efficiency in the control of locomotion to achieve endurance. The development of computer-based controllers for autonomous legged locomotion has been a topic of interest since 1970 . However, traditional gait controllers, based on leg trajectory generation and control, are not the best choice for reducing power consumption at ground contact nor during the leg phases [2].

Nature is a good source for inspiration. Studies on spinal cats, have shown that swing initiation is triggered by stretching the hip-flexor muscles, indicating that the leg is extended backwards, and it is delayed while the extensor muscles are loaded [24]. This has also been found in the locomotion of horses through observation $[25,23]$. This knowledge can motivate strategies for phase transitions in the locomotion cycle controller, as shown in Fig. 1, where a swing phase and a stance phase are preceded by toe-off and touch-down phases respectively. These four phases of locomotion, observed in nature, are very similar to those programmed in all previous developments of walking machines, where the leg motion is commanded using reference trajectories to be followed by the foot, with each trajectory matching one of the leg phases (lift the foot, transfer forward, place the foot, transfer backwards propelling the body) [2]. Thus, the main difference between the conventional and the current trend is not in the definition of leg phases but in the way of controlling the motion within each of the leg phases and the phase transition mechanisms.

Instead of following the foot trajectory until reaching the end, current trends try to exploit natural system dynamics by merging active and passive dynamics in a leg cycle, which could satisfy power requirements and energy efficiency for an agile locomotion performance [26]. In order to improve the interaction with the ground, inherent adaptable compliance is required at the

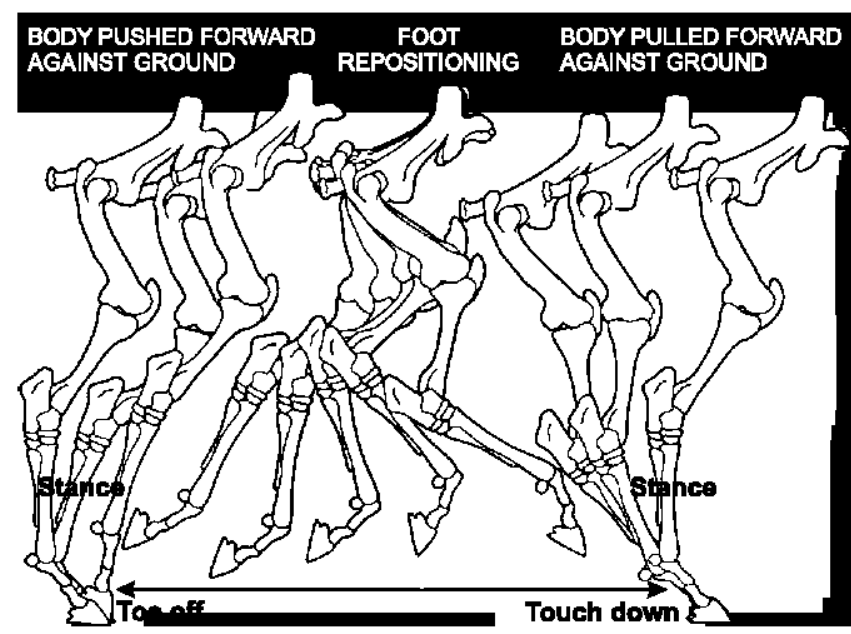

Fig. 1. Leg phases observed in the horse's right hind leg, moving through a cycle from mid stance to mid stance, based on digital video frame overlays [23]

legs [27-29]. Adjustable compliance in actuators improve energy efficiency in legged locomotion by adjusting the system's natural dynamics, so that optimal behavior can be achieved for a variety of ground stiffnesses [21].

Many researchers working on bipedal-walking robots have relied on developing heuristic controllers based on simple physical models and intuition that resemble the biological phase transition mechanism [30-32]. In fact, animals that run feature a reflexbased coordination of movements rather than centralized neural coordination [33]. Therefore, the idea is to provide the desired impulse to let the leg move, exploiting its natural dynamics until some reflex is activated, which would trigger the next phase of locomotion.

Following these principles, the locomotion controller for a robot leg has been designed. The leg called HADE (acronym of the project Hybrid Actuator Development) has been designed and developed for achieving agile locomotion. In order to develop a leg mechanism able to provide the robot with agile locomotion abilities, nature is the best source of inspiration. Guidelines have been taken from the anatomy of horses' legs which have evolved to provide strength, endurance and agility. Strength is provided by specific metal alloys featuring large payload-to-weight ratios. Endurance is achieved by means of elastic energy storage during some phases of the gait cycle, released into the system to help the actuators in the phases when large power is required. Agility is achieved by means of high-speed actuators and interaction control schemes based on active compliance. A detailed description of the leg design can be found in [34]. In this paper the control architecture for the compliant locomotion of the HADE leg is presented. Section 2 briefly describes the structural design of the HADE leg and actuation system. Then, Section 3 presents 


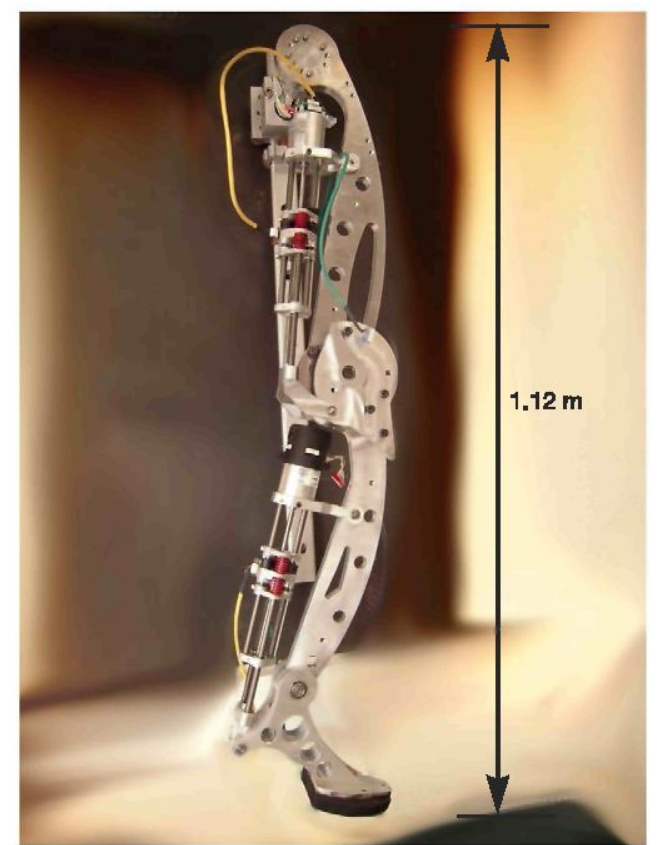

Fig. 2. First prototype of the HADE leg resembling a horse's leg.

the control architecture designed for efficient, agile locomotion. Sections $\mathbf{4}$ and 5 detail the control algorithms for the locomotion cycle and inner joint motion respectively. Finally, Section 6 shows experimental results with the real prototype and Section 7 presents some conclusions.

\section{The HADE leg for agile locomotion}

Horse's legs are adapted to give horses speed, endurance, agility and strength superior to any other animal of equal size. This adaptation is based on [35]: (1) Longer legs than similar quadrupeds relative to body size, providing longer stride lengths; (2) The horse's legs are relatively lightweight, yet strong enough to deliver very large thrusts and to sustain tremendously heavy loads; (3) The horse's hip joint is mainly a hinge to turn the thigh forward and backward. The abduction/adduction movement is practically negligible. Similarly, knee and ankle are 1-DoF joints. Thus, all the muscles and tendons focus their effort in simple joint motions; And (4) all this with enough economy of effort to provide endurance, which is achieved by means of elastic energy storage in tendons during certain phases of the locomotion cycle and the later return of this energy to the more exigent phases.

The HADE leg has been designed following these four valuable guidelines: it is a relatively lightweight long leg with three 1-DoF joints, propelled by elastic actuation. Fig. 2 shows a photograph of the HADE leg which resembles the anatomy of the horse's leg. The rest of this section will expand upon the details of leg design and actuation system selection.

\subsection{Leg design}

The design of the first prototype of the HADE leg is shown in Fig. 3. It is a planar 3-DoF leg composed of three links: thigh, shank and foot, connected through the hip, knee and ankle joints. The mechanical structure of each link has been designed in order to achieve large payload-to-weight ratio and to provide impact tolerance. The toe is endowed with a rubber pad which provides shock absorption and damping at ground contact and also increases friction between foot and ground improving horizontal propulsion.

The large payload-to-weight requirements of the structure will be achieved by manufacturing the mechanical structure using Alumec 89, a high strength aluminum alloy which undergoes a special cold stretching operation for maximum stress relief. This material is being used in the aerospace industry as it shows the best payload-to-weight properties. Table 2 shows link dimensions and masses of the first prototype, not including actuator mass, which will change for different studies. The position of center of mass and inertia tensor are referred to the joint reference frame following the Denavit-Hartenberg convention as shown in Fig. 4.

\subsection{Actuation system}

In order to determine actuator requirements for the joints of the HADE leg, joint torques and speeds from simulation have been converted to actuation requirements. To do so, transmission reduction and efficiency were considered. The requirements of actuator weight of $1.1 \mathrm{~kg}$, actuator power over 400 Watts (considering an overall actuator efficiency of 90\%), compactness and large speed are all met by brushless DC motors. Also, the
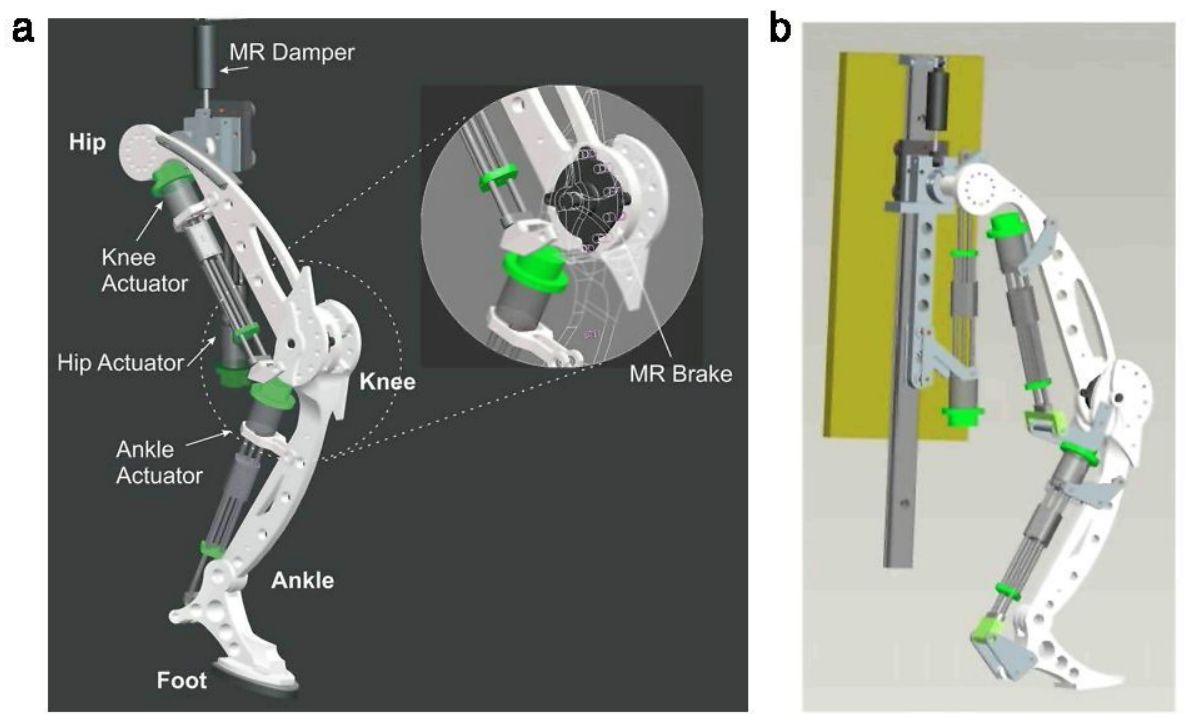

Fig. 3. Design of first prototype of the HADE leg. (a) The MR brake at the knee is showed in zoom-in view; (b) reverse view of the actuators. 
Table 2

Physical parameters of the HADE leg prototype.

\begin{tabular}{lcccc}
\hline Link & & Thigh & Shank & Foot \\
\hline Length $(\mathrm{mm})$ & & 505 & 461 & 205 \\
Mass $(\mathrm{kg})^{\mathrm{a}}$ & & 3.5 & 2.6 & 1.6 \\
& $x$ & 325 & 143 & 93.2 \\
Center of mass $(\mathrm{mm})^{\mathrm{b}}$ & $y$ & -10.8 & -16.6 & -41.1 \\
& $z$ & 35.2 & 10.1 & -0.02 \\
& $I_{x x}$ & 16.4 & 8.95 & 5.17 \\
& $I_{x y}$ & -1.87 & 1.33 & 2.61 \\
Inertia tensor $\left(10^{-3} \mathrm{kgm}^{2}\right)$ & $I_{x z}$ & -0.05 & -0.52 & 0.001 \\
& $I_{y y}$ & 1060 & 110.7 & 9.36 \\
& $I_{y z}$ & 0 & -0.01 & -0.001 \\
& $I_{z z}$ & 1050 & 108.6 & 13.48 \\
\hline
\end{tabular}

a Link masses do not include actuator mass.

b $\mathrm{CM}$ position and Inertia tensor refer to Denavit-Hartenberg joint reference frames.

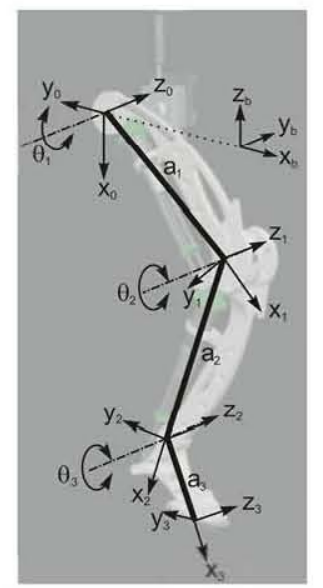

Fig. 4. Kinematic parameters of HADE leg.

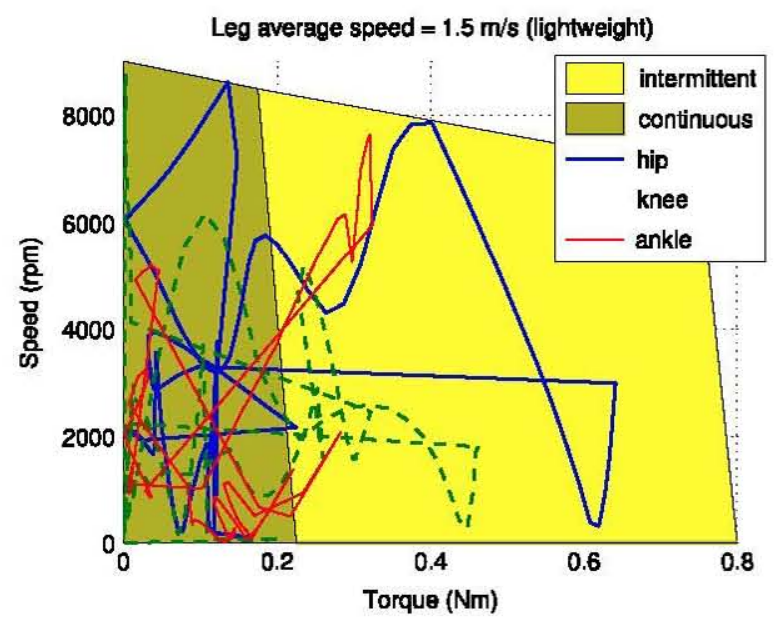

Fig. 5. Motor torque-speed diagram and joint requirements for a leg average speed of $1.5 \mathrm{~m} / \mathrm{s}$.

need for a compliance control scheme led us to use Series Elastic Actuators (SEAs). Therefore Yobotics' series elastic actuators SEA23-23 were selected.

Fig. 5 shows the speed-torque diagram of the motor used in the SEA23-23. This motor is a Moog Silencer BN23-23ZL-03LH. The shaded area represents the admissible operation points of this motor for continuous and for intermittent operation modes. Joint speed-torque trajectories obtained from the simulated locomotion cycle have been plotted over the motor characteristic diagram, considering an efficiency of $90 \%$ and the variable transmission

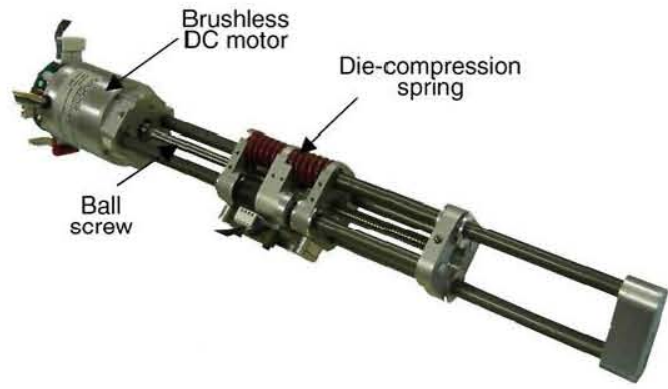

Fig. 6. Yobotics SEA23-23.

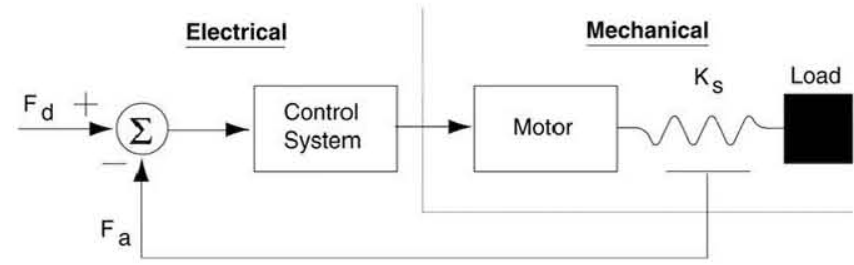

Fig. 7. Schematic diagram of a series elastic actuator [36].

reduction ratios. In order to check that the motor is able to provide the speed-torque requirements for this application, the simulated joint trajectories have been obtained for the maximum leg speed of $1.5 \mathrm{~m} / \mathrm{s}$ and a payload-to-weight ratio of 2 .

Although the lines representing joint trajectories are difficult to be followed, the relevant information from this graph is to check that all the lines fall within the admissible operation area of the motor. The diagram in Fig. 5 shows that most of the joint trajectories fall within the continuous operation area, while some part of the joint trajectories fall within the intermittent operation range of the motor. Considering that the locomotion cycle of the leg makes the motors work intermittently and also that the duration of the large power spans is almost instantaneous, lasting less than $50 \mathrm{~ms}$, it is worthily assumed that it is supported by intermittent motor operation.

Added to the power requirements described above, the compliant interaction with the environment can only be met by using series elasticity between the motor and the joint. SEAs are a family of actuators specifically designed for force control in robotic systems. They are backdriveable, compliant actuators with resistance to impact and vibration. The power-to-weight ratio of this actuator is almost $600 \mathrm{~W} / \mathrm{kg}$. SEAs are high force-to-weight, high power-to-weight actuators, and they are the precursors of a family of compliant actuators with a novel mechanical design architecture that goes against the common machine design principle of "stiffer is better" $[36,27]$. They are composed of a motor, a transmission and a load, but they have an elastic element connected in series between the transmission and the load. Fig. 6 shows a photograph of the Yobotics SEA23-23 and Fig. 7 shows a simple diagram of a general SEA. A detailed description of the SEA design and performance has been already published by their inventors $[37,36]$ and falls out of the scope of this paper.

In SEAs, stiff load cells are replaced with a compliant element which enables the indirect measurement of joint forces by measuring the deflection of the spring. In addition, the elastic element gives the actuator low output mechanical impedance, in contrast with traditional actuators with high power-to-weight ratio which usually have high output impedance.

The motor used in SEAs features good position accuracy to give a good force output. The better the motor can modulate the spring position, the cleaner the force output of the spring. Besides, the elastic element filters noise and allows for an increase in the force controller gain within stable operation. As a whole, SEAs improve conventional actuators in force control response. 
Table 3

Actuator specifications.

\begin{tabular}{|c|c|c|}
\hline Joint & Hip/Ankle & Knee \\
\hline Model & SEA23-23 & SEA23-23 (modified) \\
\hline Weight (kg) & 1.1 & \\
\hline Diameter (mm) & 58 & \\
\hline Length (mm) & $150+$ strok & \\
\hline Maximum Stroke (mm) & 150 & \\
\hline Ball-screw pitch (mm) & 2 & 5 \\
\hline Motor & \multicolumn{2}{|c|}{ Moog Silencer BN23-23ZL-03LH } \\
\hline Rotor inertia (gcm2) & \multicolumn{2}{|c|}{106} \\
\hline Operating voltage (V) & \multicolumn{2}{|l|}{48} \\
\hline Maximum current $(A)$ & \multicolumn{2}{|l|}{20} \\
\hline Maximum speed (m/s) & 0.27 & 0.67 \\
\hline Cont. force at max. speed $(\mathrm{N})$ & 566 & 226.4 \\
\hline Continuous power (W) & 166 & 166 \\
\hline Int. force at max. speed (N) & 1300 & 520 \\
\hline Intermittent power $(W)$ & \multicolumn{2}{|l|}{629} \\
\hline Power-to-weight ( $\mathrm{W} / \mathrm{kg})$ & \multicolumn{2}{|l|}{571} \\
\hline Closed-loop bandwidth (Hz) & \multicolumn{2}{|l|}{35} \\
\hline Saturation bandwidth (Hz) & \multicolumn{2}{|l|}{7.5} \\
\hline Efficiency (\%) & \multicolumn{2}{|c|}{90} \\
\hline Spring & \multicolumn{2}{|c|}{ Century die spring 1222-A } \\
\hline Spring stiffness ( $\mathrm{N} / \mathrm{mm})$ & \multicolumn{2}{|c|}{$4 \times 78.4$} \\
\hline Encoder & \multicolumn{2}{|c|}{ USDigital EM1-0-500 } \\
\hline Pos. resolution (lines per mm) & \multicolumn{2}{|c|}{19.68} \\
\hline
\end{tabular}

All actuation requirements stated above 2.2 are met by the Yobotics SEA23-23 shown in Fig. 6 for hip and ankle joints. Knee requirements are met by the same actuator using a $5 \mathrm{~mm}$ lead ball screw. Table 3 lists actuator specifications for the three joints of the HADE leg.

\subsection{Damping properties}

Series elasticity provides good force control at the joints and adds some shock tolerance to the mechanism. Also, it decouples the motor inertia from the load, so that slight position errors will be absorbed by the elastic element, preventing the control system from reacting to unexpected impacts. These properties largely benefit the adaptation to the ground in high-speed locomotion. However SEA is not enough. As will be detailed in Section 4, along the leg cycle variable damping is required at the knee. A LORD RD2087-01 magneto-rheological (MR) rotary brake has been placed at the knee to test the efficiency of variable damping along the gait cycle (see Fig. 8(a)). Magneto-rheological brakes and dampers are resistive actuators based on magneto-rheological fluids, a kind of smart material of the magnetoactive family [38]. The MR brake is designed as shown in Fig. 8(b): An outer housing contains a rotor joined to the knee axis. Between the rotor and the housing the MR fluid interferes. As a magnetic field is applied to the MR fluid, the rheological characteristics of the fluid change to provide increased viscosity. The viscosity of the fluid influence torque that brakes the rotor, thus providing controllable viscous rotary damping [39]. Fig. 3 shows the integration of the MR brake inside the knee of the HADE leg.

\section{Control architecture}

The control architecture designed for the motion control of the HADE leg is shown in the block diagram of Fig. 9. It is a conventional cascade controller, with three inner joint controllers (one for each joint) and one outer loop to control the locomotion cycle in the Cartesian leg space and phase transition. Analyzing the block diagram in the figure from upper level to lower level control, the first block found is the locomotion cycle controller, which has been shaded in dark gray. Inputs to the locomotion controller are: desired cycle speed which matches the desired average speed of the leg; vertical ground reaction force, measured from the load cell at the foot pad and; foot Cartesian position, measured from joint a

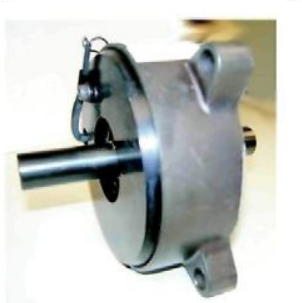

b

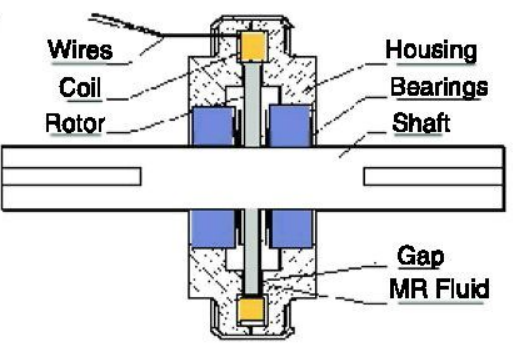

Fig. 8. (a) LORD RD-2087-01 magneto-rheological rotary brake; (b) mounting scheme.

encoders and converted using direct kinematics. The locomotion cycle controller will decide on the next action to be taken in the leg task space as a function of the leg's current state and the phase transition, as will be detailed in Section 4. As a result, two vectors come out from the locomotion cycle controller, which are the next desired foot position $\mathbf{x}\left(\mathbf{x}_{0}, \mathbf{y}_{0}\right)$ and desired force at the foot $\mathbf{F}\left(\mathbf{F}_{\mathbf{x}_{0}}, \mathbf{F}_{\mathbf{y}_{0}}, \mathbf{M}_{\mathbf{z}_{0}}\right)$, both in the leg's base reference frame (see Fig. 4). Both vectors are then converted to joint space as joint position and torque references to the three parallel joint controllers. These three low level controllers, shaded in light gray, make sure that each actuator motion follows the commands from the upper level locomotion cycle controller. Two different control schemes have been designed and tested as joint controllers: a direct force controller and an active compliance controller. Both schemes are described in Section 5. After actuating the joints, the resulting change in the leg state is acquired by the force and position encoders at the actuator, which are fed back to the joint controllers and locomotion cycle controller through the appropriate kinematic transforms.

The above described control architecture has been programmed using LabView and it runs on an National Instruments PXI-1042Q platform using a NI PXI-8106RT controller (Core 2 Duo $2.16 \mathrm{GHz}$ processor with RealTime embedded OS). The inner joint control loop is performed at $10 \mathrm{kHz}$ while the outer locomotion cycle controller runs every $5 \mathrm{~ms}$.

While the majority of previous works focused the controller design on the trajectory control of robot motion, some researchers have been considering exploiting inherent dynamics making use of robot force control approaches [26]. The goal of achieving agile locomotion led us to a similar design focused on exploiting inherent dynamics with the main goal of improving energy efficiency. This has been achieved by the combined use of two controllers, one for the locomotion cycle, inspired in previous works that use bio-inspired phases of locomotion and state transitions, and one controller for the joint motion, which is based on impedance control. Previous bio-inspired locomotion controllers use an inner direct force controller for joint motion. A joint impedance controller allows to provide a real spring-damper behavior with variable stiffness and damping coefficients. Therefore, both controllers rely on the force control of locomotion rather than trajectory control, in such a way that the inherent dynamics like inertia, damping and compliance help the actuators to propel and brake the structure along the locomotion cycle. Thus, within this scheme, actuators apply power only when it is needed, contrarily to trajectory-control schemes, and the negative mechanical work is reduced and even avoided at the actuators, making use of a special mechanical device used for the active damping of the knee, the MR rotary brake described in Section 2.3.

\section{Locomotion cycle controller and phase transition}

The locomotion cycle controller defines phase states and transitions for one locomotion cycle of the HADE leg. It has been 


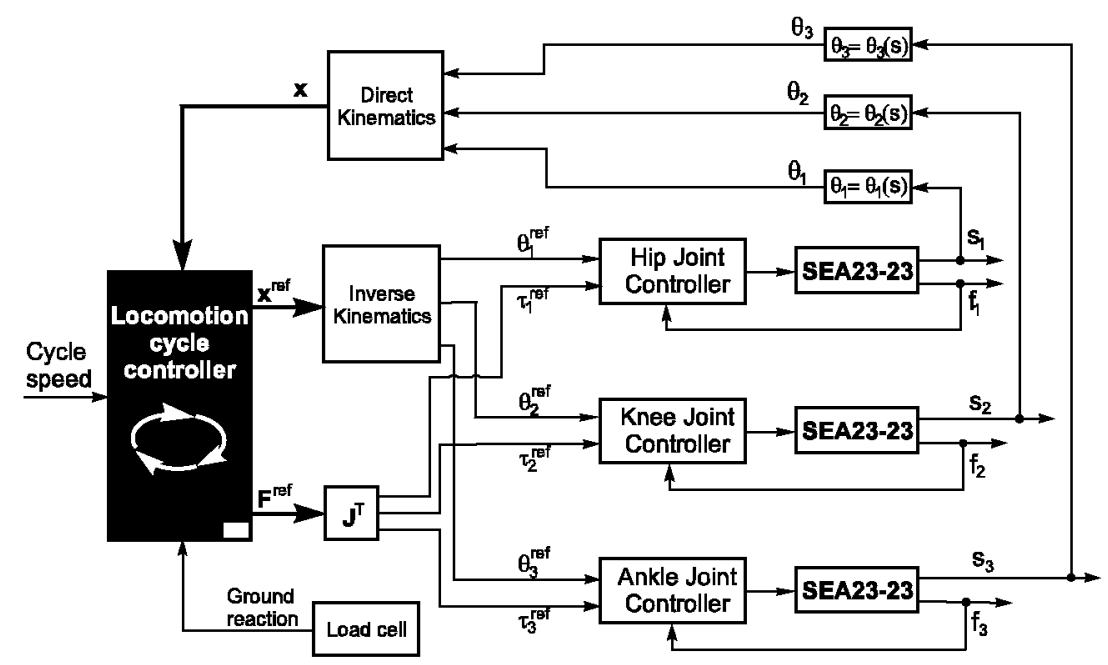

Fig. 9. Control architecture for the locomotion of the HADE leg.

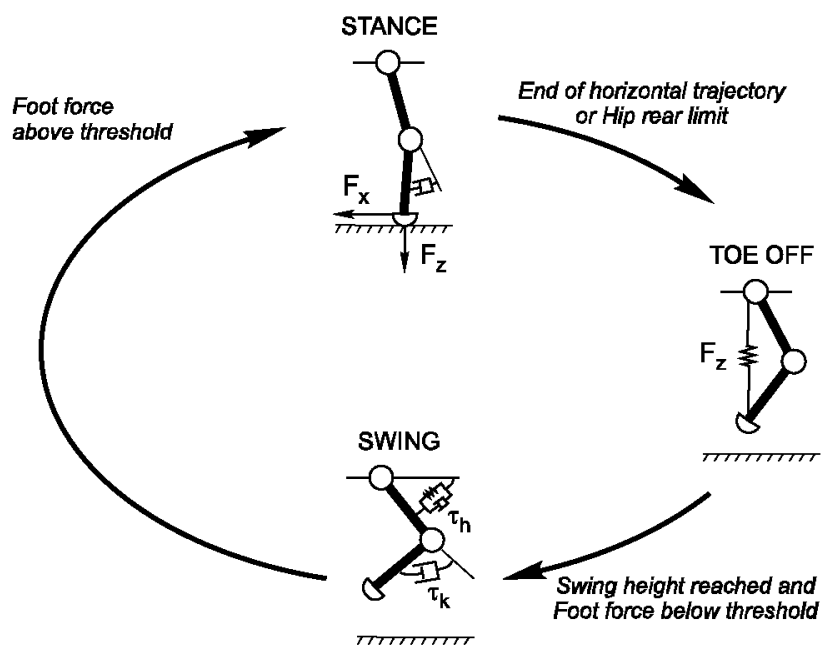

Fig. 10. Control states of a locomotion cycle.

designed as a state machine with three states matching three locomotion phases: stance, toe-off and swing, shown in Fig. 10.

STANCE The stance phase is intended to propel the body forward through foot-ground interaction. From the instant of touch down to mid stance the foot pulls the ground in the horizontal direction while after mid stance the foot pushes the ground in the horizontal direction (see Fig. 1). Mid stance occurs when the foot trajectory reaches the horizontal projection of the hip on the ground. Therefore, while the vertical foot-ground interaction force is nearly constant supporting the body weight, the horizontal component helps the foot to follow a linear trajectory. Finally, the foot pitch moment is computed so that the foot is kept parallel to the ground to maintain the largest friction area along the stance phase.

Let us define the body reference frame $\left\{x_{b}, y_{b}, z_{b}\right\}$ as a Cartesian reference frame parallel to the world's reference frame whose origin coincides with the leg base reference frame $\left\{x_{0}, y_{0}, z_{0}\right\}$ (see Fig. 4), so that the following transformation holds:

$\left(\begin{array}{l}x_{b} \\ y_{b} \\ z_{b}\end{array}\right)=\left(\begin{array}{ccc}0 & -1 & 0 \\ 0 & 0 & 1 \\ -1 & 0 & 0\end{array}\right) \cdot\left(\begin{array}{l}x_{0} \\ y_{0} \\ z_{0}\end{array}\right)$

All forces and positions of the foot in the locomotion controller are considered in the body reference frame and are converted to the leg base reference frame afterward. In order to achieve
Table 4

Locomotion cycle controller gains.

\begin{tabular}{lllll}
\hline STANCE & & & & \\
$K_{x}^{S T}$ & $B_{x}^{S T}$ & $K_{z}^{S T}$ & $K_{\phi}^{S T}$ & $B_{2}^{S T}$ \\
100 & 50 & 0.2 & 2 & 1.45 \\
\hline TOE-OFF & & & & \\
$K_{x}^{T 0}$ & & $K_{z}^{T 0}$ & $K_{\phi}^{\text {To }}$ & \\
150 & 550 & 2 & \\
\hline SWING & & & & \\
$K_{1}^{S W}$ & $B_{1}^{S W}$ & $B_{2}^{S W}$ & $B_{3}^{S w}$ & \\
80 & 50 & 0.9 & 2 & \\
\hline
\end{tabular}

the desired foot behavior described above, desired foot-ground interaction forces are computed as: a spring-damper system in the horizontal direction, using the reference foot speed and position as equilibrium points for the virtual damper and spring respectively; as a direct proportional force controller in the vertical direction, to ensure the leg supports the robot weight; and as a torsional spring in the foot pitch joint, using the reference pitch angle as equilibrium point. Thus, foot-ground interaction forces are the following:

$$
\begin{aligned}
& F_{x_{b}}^{r e f}=K_{x}^{S T}\left(-\frac{S}{2}-x_{b}\right)+B_{x}^{S T}\left(-v_{S T}^{\text {des }}-\dot{x}_{b}\right) \\
& F_{z_{b}}^{r e f}=-M g-K_{z}^{S T}\left(M g-F_{z_{b}}\right) \\
& M_{y_{b}}^{r e f}=K_{\phi}^{S T}\left(\phi^{\text {des }}-\phi\right)
\end{aligned}
$$

where $S$ is the stride length, $x_{b}$ is the current foot horizontal position, $v_{S T}^{\text {des }}$ is the reference stance speed (which we assume to be equal to the cycle speed), and $K_{x}^{S T}, B_{x}^{S T}, K_{z}^{S T}$ and $K_{\phi}^{S T}$ are the controller gains which are listed in Table 4. Please, note that, as the foot pushes the ground to propel the body forward in the horizontal motion, a negative reference speed has been used for the computation of the virtual damping component of the desired horizontal force $F_{x_{b}}$. Desired vertical force $F_{z_{b}}$ is computed from the robot weight and payload, tuned by the measured ground reaction force. $\phi^{\text {des }}$ and $\phi$ are foot desired pitch angle and current pitch angle respectively. The desired pitch angle is the one that keeps the pad parallel to the ground, which corresponds to $\phi=-0.9$ rads.

As can be observed from (3), the commanded horizontal force is a function of the foot trajectory in the horizontal direction. Therefore, during stance phase, a hybrid position force scheme is followed, where the horizontal motion is controlled based on a position reference and the vertical direction follows a direct force reference. However, although the horizontal motion 
is commanded by position, this position is converted to a commanded force proportional to the distance to be traveled by the foot, and afterward, this commanded force is converted to commanded torques at the joints which are followed by three force-based joint controllers (see Fig. 9). Therefore, the position reference does not yield a rigid foot trajectory. If the foot trajectory is interrupted by any obstacle, the force on the obstacle will be constant until the obstacle gets out of the way, and then the foot will continue its motion naturally. In the case of a conventional trajectory following, the presence of an obstacle will stop the motion but the reference position will continue to increase, so the force applied to the obstacle will increase accordingly: such a situation is hazardous for the obstacle and for the robot itself.

To achieve a natural stance, a damping torque is needed at the knee, so the final reference torque at the knee during stance is as follows:

$\tau_{2}=\tau_{2}^{\text {act }}-B_{2}^{S T} \dot{\theta}_{2}$

where $\tau_{2}^{\text {act }}$ is the actuated torque provided by the actuator and $\tau_{2}$ is the resultant torque at joint 2 after adding some damping. Note that $\tau_{2}^{\text {act }}$ is computed from the Cartesian desired forces at the foot following Eqs. (3)-(5) through inverse dynamics.

TOE-OFF The condition for state transition from support to toe-off is the end of the horizontal trajectory or a rear limit on hip angle. During toe off, the foot lifts off the ground by means of a vertical force pulling the foot upwards as shown in Fig. 10 and a horizontal force pulling the foot toward the hip horizontal component. Thus, foot forces at this state are given by:

$F_{x_{b}}^{\text {ref }}=-K_{x}^{T 0} x_{b}$

$F_{z_{b}}^{\text {ref }}=K_{z}^{T 0}\left(z^{\text {des }}-z_{b}\right)$

$M_{y_{b}}^{\text {ref }}=K_{\phi}^{\text {To }}\left(\phi^{\text {des }}-\phi\right)$

where $z^{\text {des }}$ is the desired foot vertical position at the beginning of swing and $z_{b}$ is the current foot vertical position. The desired increment in foot height is $6 \mathrm{~cm}$. The foot pitch angle could be left uncontrolled, however some position control has been performed in order to improve ground clearance.

SWING The controller shifts from toe-off to swing state when the ground reaction force $F_{z_{b}}$ at toe-off equals 0 and the desired swing height has been reached. Using both conditions allows for the avoidance of terrain obstacles during toe-off, so a larger initial swing height will be allowed if the ground reaction force is still positive. Once the desired swing height is reached, the leg motion is propelled at the hip, while the knee and ankle are allowed to move freely exploiting the leg inertia. Therefore during swing phase, desired hip joint torques are modeled as a spring-damper system, trying to propel the hip toward its front limit. Meanwhile, knee and ankle are simply damped:

$\tau_{1}^{s w}=K_{1}^{S W}\left(\theta_{1}^{f w}-\theta_{1}\right)+B_{1}^{s W}\left(\dot{\theta}_{1}^{\text {des }}-\dot{\theta}_{1}\right)$

$\tau_{2}^{S W}=-B_{2}^{S W} \dot{\theta}_{2}$

$\tau_{3}^{s w}=-B_{3}^{s w} \dot{\theta}_{3}$.

Notice that the commanded torque at the hip is modeled as a torsional spring-damper attached to a hip forward limit position. Therefore, as long as the hip joint approaches this limit point, the commanded torque at the hip is reduced. Therefore, near the hip limit position, the foot will start a natural downward motion, caused by a reduction on hip torque and the damping at the knee, yielding a natural touch down without additional locomotion phases, just by exploiting inherent dynamics. The controller changes from swing to stance when the vertical groundcontact force reaches $10 \mathrm{~N}$.

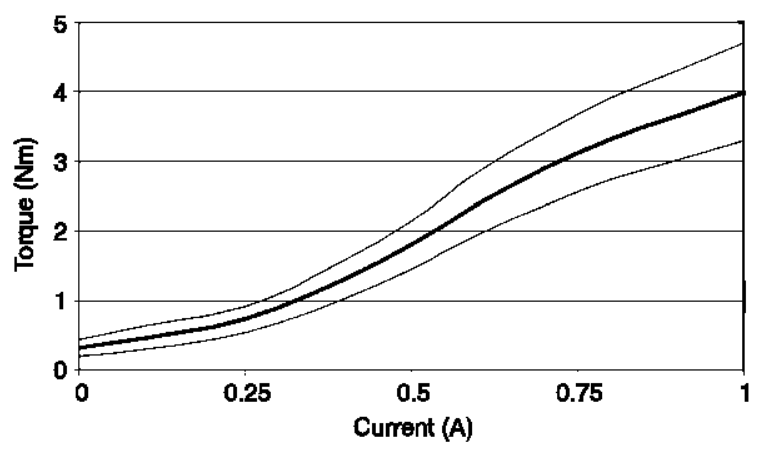

Fig. 11. Typical torque-current curve of the LORD RD-2087-01 MR brake.

Such a command of joint motion allows for a reduction of power requirements at the knee and the ankle during swing. However, actively damping the joints also wastes actuator power, which is commanded to brake the joint. Some passive damping device could help save energy. However, note that the locomotion controller proposed herein imposes damping at the knee during different phases of the locomotion cycle and that the damping coefficient is not the same, but varies from one phase to the other. Therefore, joint damping is not constant but should be adapted to the different phases of the cycle. Consequently, an active damping system is needed for successful natural locomotion, but the energy consumed by the actuators during the damping motion must be reduced.

In this project, a LORD RD-2087-01 magneto-rheological rotary brake has been placed at the knee to actively control knee damping along the gait cycle. As a magnetic field is applied to the MR fluid inside the brake, the characteristics of the MR fluid inside the device change to provide increased resistive torque output with practically infinite precision and an under $10-\mathrm{ms}$ response time. The maximum power consumption of the MR brake is $1 \mathrm{~A}$ at 12 VDC for a maximum resistive torque of $4 \mathrm{Nm}$. Fig. 11 shows the typical torque-current curve for the MR rotary brake.

Using the MR rotary brake, the SEAs are used for active motion, while passive damping is performed by the MR brake. Notice that the MRF brake is not intended to lock the knee but to impose a resistive damping torque, which can be actively modified along the different phases of the locomotion cycle. The controller gains have been tuned manually to achieve natural-looking motion. Table 4 shows gains for the HADE leg controller. Gains $B_{2}^{S T}$ and $B_{2}^{s w}$ are controlled through the MR rotary brake.

\section{Joint control schemes}

Two control schemes have been developed for the HADE leg joint motion. The first one is a direct force controller acting on the SEA at each joint. Once again inspired by biology, we tried to add some compliance to the joints. The motivation originated mainly from using inherent elasticity to achieve stable behavior during hard contact, protecting the joints from impact shock and storing elastic energy for energy efficient motions. We initially considered the use of the inherent elasticity of SEAs; however, after some attempts we found out that the spring in a SEA was too stiff to be used as an elastic energy storage unit: it is just a low-impedance transductor. Therefore, our second attempt was to develop an active compliance controller at the joints. Both control schemes are presented in this section.

\subsection{Direct joint force control}

A preliminary joint controller version for the HADE leg is a direct force controller acting on the SEA at each joint. The force 


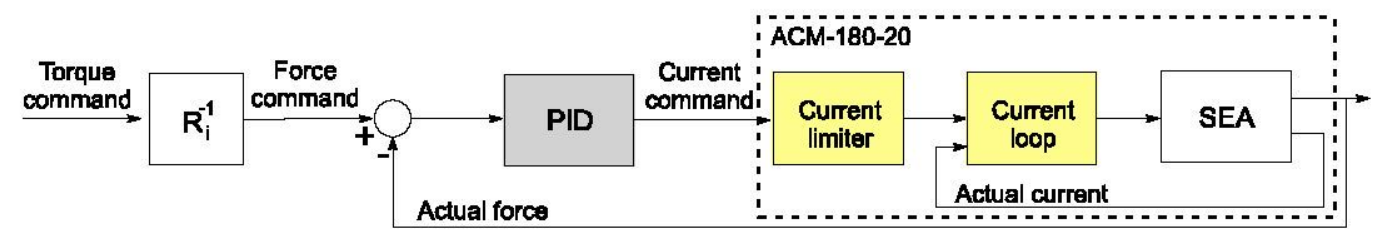

Fig. 12. Joint force control based on PID.

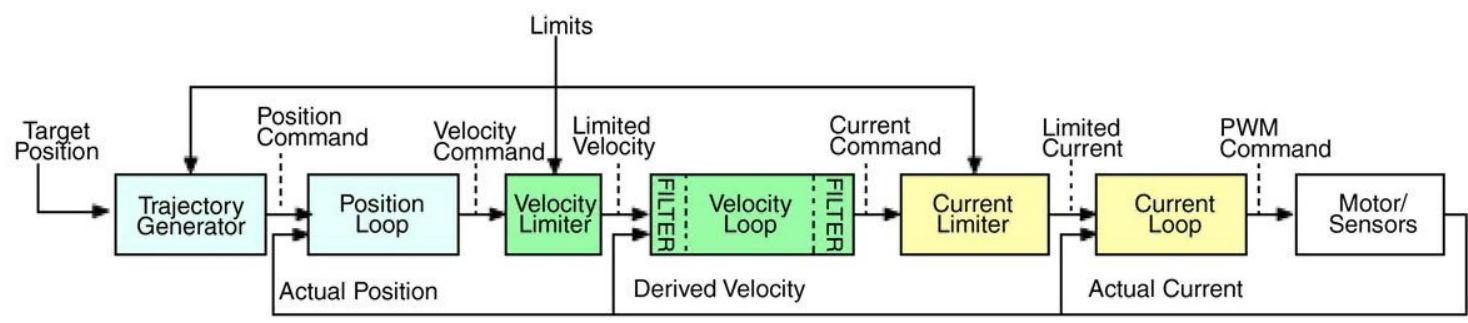

Fig. 13. Cascade controller of the Accelnet Module ACM-180-20.

controller is based on conventional PID control acting directly on the current command of the SEA's power amplifier. Fig. 12 shows a block diagram of the control scheme.

The SEA's power amplifier is an Accelnet Module ACM-180-20. For the control of DC brushless motors, the Accelnet Module can be used in one of these three operation modes: (1) position, (2) velocity, and (3) current command. The implementation of these three operation modes is based on the cascade controller shown in Fig. 13. In the block diagram of this cascade controller, the inner loop is a current PI feedback controller. The closest outer loop to the current loop is a PI velocity controller. Finally, the most outer loop is a position $P$ controller. The gains of the three controllers are programmable. The controllers are turned on and off depending on each operation mode. For the current command operation mode, the position and velocity loops are switched off. For the velocity command operation mode, only the position loop is switched off. Finally, for the position command operation mode, all three controllers operate simultaneously.

In the joint force controller shown in Fig. 12, the Accelnet Module is used in current command operation mode. This allows for some flexibility when designing specific controllers.

\subsection{Active compliance joint control}

The preliminary joint controller based on the direct force control scheme described above was not compliant enough for smooth and safe operation. In order to improve joint compliance, an active compliance joint controller was proposed.

Unlike to traditional impedance control schemes which use an inner position or velocity loop and outer force loop to control the mechanical impedance $[40,41]$, the control scheme used for this work implements an inner force loop with an outer position loop. Previous attempts to implement real impedance control following this scheme failed due to the inadequate actuation system. Conventional electro-mechanical and hydraulic actuators are very good at position control, but poor at force control due to noise and nonlinearities. That is why impedance control schemes usually rely on inner position loops, which filter the force noise. In this work we were able to implement an inner force loop impedance control and this was possible thanks to the use of a SEA which allows for direct force control.

A compliance control scheme was implemented. Compliance control emulates the behavior of a spring-damper system, which models mammalian muscle behavior quite well [42]. The block diagram of the control scheme is shown in Fig. 14, where $G_{z}$ is

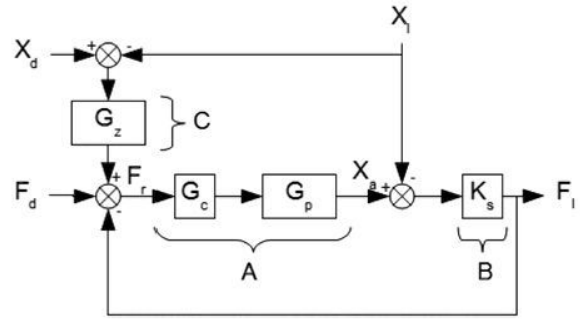

Fig. 14. Joint compliance control scheme block diagram.

the model corresponding to a spring-damper system, $G_{c}$ is the transfer function of the inner loop controller, $G_{p}$ is the actuator's transfer function, $K_{\mathrm{s}}$ is the die compression spring constant, $F_{\mathrm{d}}, F_{l}$ and $F_{r}$ are the desired force, exerted force, and the force reference respectively. Finally, $X_{d}, X_{l}$ and $X_{a}$ are the desired position, the load position and the actuator position. Parting from the diagram shown in Fig. 14, making $X_{d}$ and $F_{d}$ equal to zero, the mechanical impedance of the actuator can be derived as follows:

$Z=\frac{F_{l}}{X_{l}}=-\left(\frac{A B}{1+A B}\right)\left(\frac{1}{A}+C\right)$.

Since $A B \gg 1$ in the controlled bandwidth of the system:

$Z=-\left(\frac{1}{A}+C\right)$

The term $A=G_{c} G_{p}$ has usually high gain in the controlled bandwidth, since $G_{c}$ implements an integral action. As a consequence $Z \approx C$. This statement is correct except near to the cutoff frequency, $\omega_{c}$, which is defined as $\left|A\left(j \omega_{c}\right)\right|=1$ and is approximately related to the response time, $\pi=\omega_{\mathrm{c}} t_{\text {response. }}$. In biomimetic applications, such as agile robots and exoskeletons, this is important because the actuator needs to emulate the actual behavior of its biological counterparts and we have already mentioned that they act as spring-damper systems.

In order to obtain mechanical impedance spring-damper behavior we have chosen $C=K(1+b s)$. Fig. 15(a) shows a Bode diagram of the system behavior (thin line) overlapped with an ideal spring-damper (thick line) system, both simulated using MATLAB. This figure shows that a controlled actuator cannot imitate perfectly any desired physical impedance. The thin line shows the frequency response of the controlled actuator trying to achieve a spring-damper physical impedance. By comparing with the simulated physical spring-damper one can see that the 

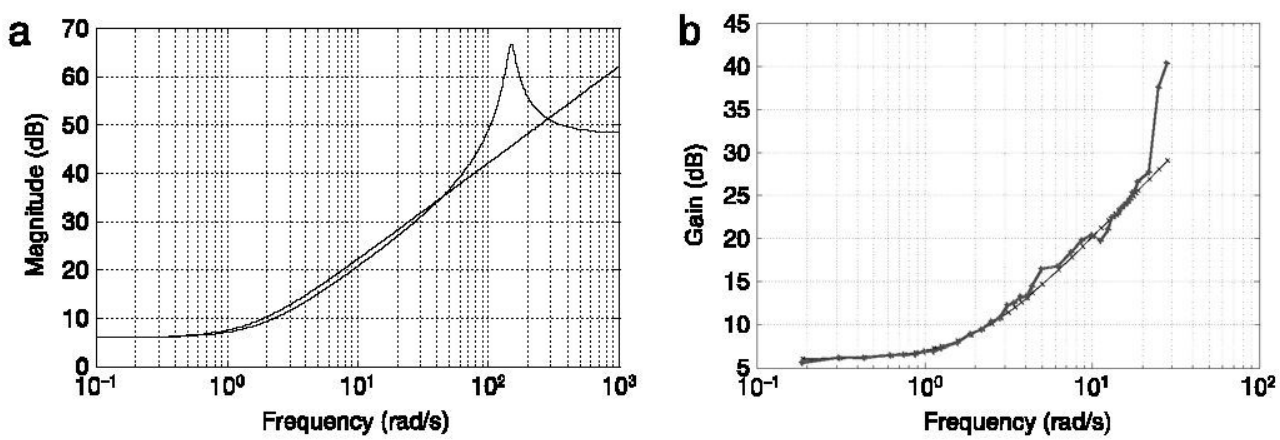

Fig. 15. (a) Bode diagram of the theoretical actuator impedance (thin line) overlapped with the ideal spring-damper system (thick line). (b) Bode diagram for the actuator impedance control with $k=2$ and $b=0.5$. Experimental results are shown by the thick line, while theoretical values are shown by the thin line.

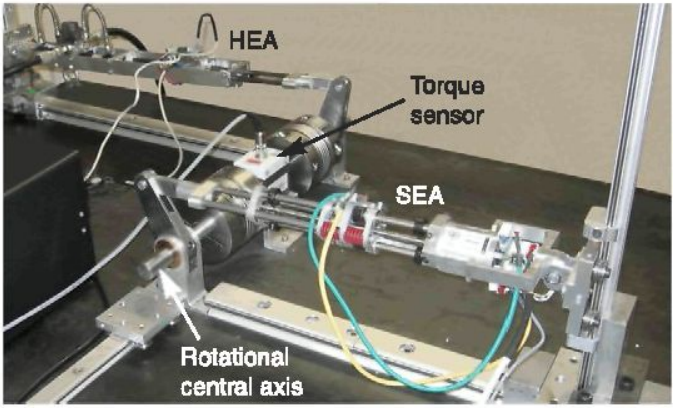

Fig. 16. Actuator test bench used for obtaining the mechanical impedance of the compliance controlled SEA.

controller can only imitate the desired dynamic behavior inside its response bandwidth, in this case $\mathbf{4 0} \mathrm{rad} / \mathrm{s}$. Therefore, in the range $[0 \mathrm{rad} / \mathrm{s}, 40 \mathrm{rad} / \mathrm{s}]$ the controller allows the actuator to behave like a spring-damper. For frequencies above $40 \mathrm{rad} / \mathrm{s}$ the actuator response matches the mechanical structure of the actuator. Concretely the constant gain that the system response approaches to for high frequencies matches the stiffness coefficient of the spring in the SEA. Therefore at high frequencies the controlled actuator behaves like a spring, just as predicted by [37].

\section{Experimental results}

Four experiments have been carried out in order to assess the performance of the proposed control schemes. First, a validation test was performed in order to analyze whether the compliant actuation system is really behaving like a spring-damper system with the proposed compliant joint controller. For this purpose, the Bode diagram of the system's mechanical impedance is obtained and compared with the corresponding spring-damper model. In order to avoid perturbing effects from the load, one of the actuators was removed from the leg and placed in an actuator test bench, shown in Fig. 16. In this test bench, the SEA is connected to a rotational central axis (simulating a hinge joint) while an antagonist-load hydro-elastic actuator (HEA) opposes the SEA motion by exerting programmed loads.

In order to obtain the Bode diagram of the system's mechanical impedance, the position of the SEA was externally modified by the antagonist actuator and the contact force measured. Initially, the force and position reference signals were set to zero. Then the antagonist HEA changed the load position $X_{l}$ sinusoidally at different frequencies. Afterward, the load position and the force exerted on the load were measured and their amplitudes divided in order to get the mechanical impedance of the system. Then, the experimental mechanical impedance was compared with the theoretical one. The results are shown in Fig. 15(b), which compares the impedance obtained experimentally for $K=2$ and $b=0.5$ with the equivalent spring-damper system. Fig. 15(a) and (b) show very similar behavior for frequencies below $35 \mathrm{~Hz}$, which shows that the real controlled actuator behaves like the simulated one. As shown, the frequency range where the actuator behaves as a spring-damper system is limited by the force closedloop bandwidth $(35 \mathrm{~Hz})$.

A second test carried out aimed to show the improvement of a inner force impedance controller compared to the inner position impedance controller. For this purpose, the step response of both control schemes was compared. In order to do so, the results of a prior position-based impedance control carried out on the legs of the SILO4 robot were used [43]. The joints of the SILO4 robot are also 1-DoF rotary joints actuated by DC motors. Piezoelectric force sensors at the foot are used for feeding back the measured force to the impedance controller. Fig. 17(a) shows the step response of the controller in [43], while Fig. 17(b) shows the step response of the controller implemented in this work using the SEAs.

As these experimental results show, the use of an inner force loop results in a faster response, reduced settling time and a
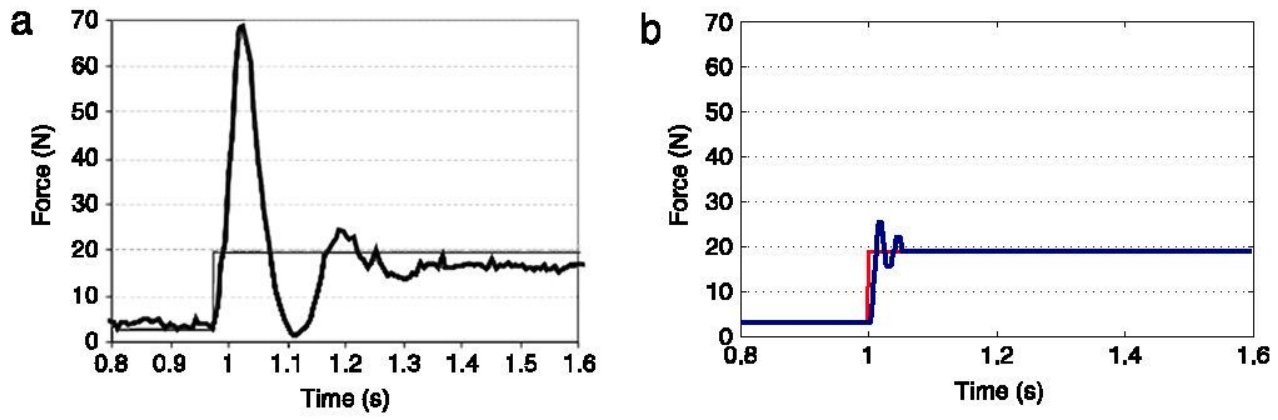

Fig. 17. Step response of an impedance control (a) using inner position loop; (b) using inner force loop. Reference command is shown by the thin line and actuator response by the thick line. 


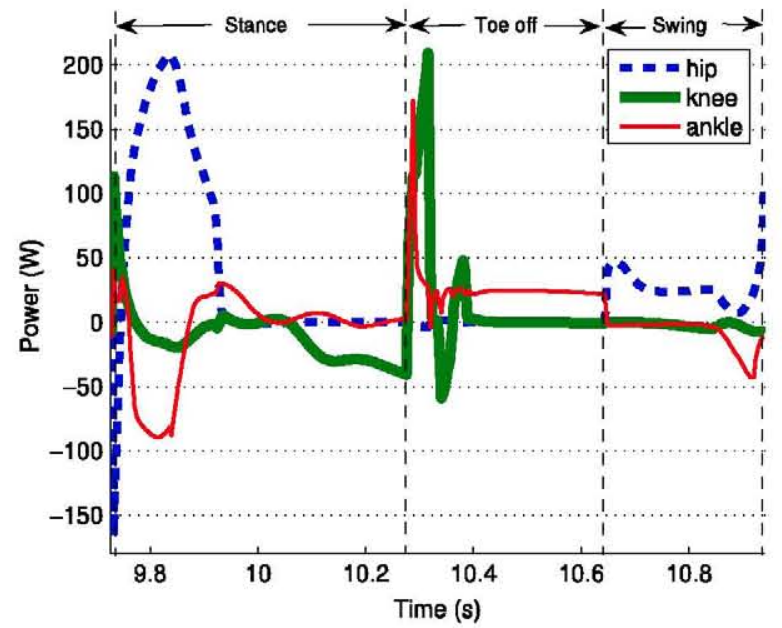

Fig. 18. Mechanical power used at the joints of the HADE leg during one locomotion cycle.

reduction of the steady state error. All these improvements are key for interaction control for safety reasons.

A third experiment has been conducted in order to experimentally measure the energy efficiency of the control architecture using the proposed combination of SEAs and MRF damping at the knee during agile locomotion. Fig. 18 shows mechanical power used for the joint motion in one leg cycle. Positive values correspond to positive mechanical work while negative values correspond to negative mechanical work, that is, braking action. This figure shows experimentally what is broadly known from biomechanics of locomotion [44], that the knee joint is mostly dissipating energy during the gait cycle. This is not the case of hip and ankle joints. Hip and ankle show percentages of the gait cycle of positive mechanical power and percentages of negative power. Just using some means of elastic energy recovery at these joints would minimize the losses (as it has been already proposed in some passive dynamic bipeds [45]). However, there are no means to help the knee to save energy. Therefore, the largest source of energy loss during walking is at the knee. Although the best solution to improve energy efficiency would be to eliminate the amount of energy dissipated by the knee, it is obvious that this is not possible because the knee has to brake the inertia of shank and foot. Therefore, any means to reduce the energy loss during the knee motion would be important.

We have proposed a control architecture that allows the leg to exploit its natural dynamics. This results in a leg motion that does not waste mechanical energy during the swing phase but at the hip. As Fig. 18 shows, the power consumed during the swing phase is almost negligible for knee and ankle actuators due to the use of inherent leg dynamics for the motion during this phase in the proposed controller. The hip however requires some energy to move the leg inertia forward in the air. Conversely, during the support phase, the propulsion of the leg while supporting the leg weight yields large power consumption. The knee consumes energy (negative power) during most of the stance phase. Therefore, the energy losses arise at the knee during stance.

Fig. 19 shows the mechanical power used at the knee during the stance phase. It shows a significant reduction in mechanical power when an external MR damper is used. The instantaneous power required for braking the knee has been reduced to one half of the power used for actively damping the knee using SEA active control. The average power required at the knee for braking during the support phase is reduced from $21.7 \mathrm{~W}$ to $15.5 \mathrm{~W}$, which means a $30 \%$ reduction in average power wasted.

The above analysis has been performed by computing the mechanical power by means of multiplying torque and velocity at

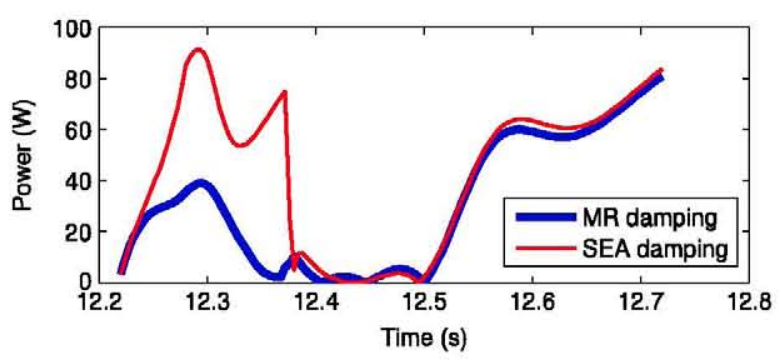

Fig. 19. Comparison of mechanical power required at the knee of the HADE leg during the stance phase when the knee is damped by active control of SEA and when the knee is damped using a MR rotary brake at the knee. The maximum instantaneous power required at the actuator is reduced by $50 \%$.

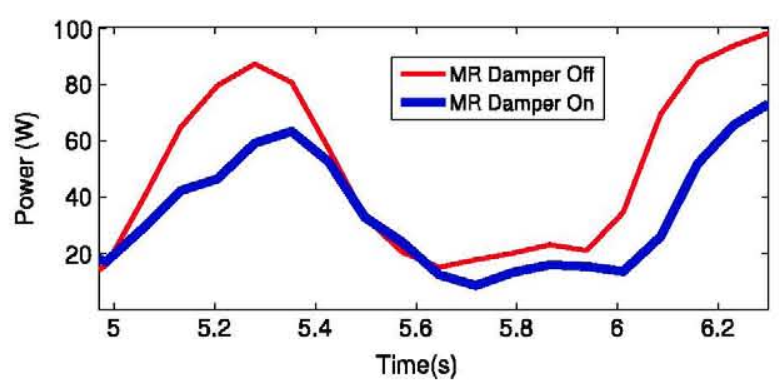

Fig. 20. Comparison of electrical power consumed by the knee SEA during a leg stance phase. The phase consists in knee extension (half of a cycle) followed by knee flexion (half of a cycle). The electrical consumption when the knee is damped by active control of SEA and when the knee is damped using a MR rotary brake at the knee is shown. The maximum instantaneous electrical power consumption is reduced by $40 \%$, not considering the power consumed by the MRF damper.

the joint, however, for a robotic application like this, the electrical power consumption is of primarily interest. Therefore, in order to assess the energy saved by the combined use of SEA and MRF at the knee, the electric power consumed by the knee SEA has been measured when the leg is in support phase in cyclic extension-flexion motions at $1 \mathrm{~Hz}$ frequency. The leg has been added a $12 \mathrm{~kg}$ load over the hip which corresponds to the robot's body weight supported by the leg in support phase. The MRF damper is activated at maximum power during the whole support phase. A video of this experiment can be found in [46]. Fig. 20 compares the electrical power consumed by the knee SEA in a support cycle when the MRF damper is activated, and when the MRF damper is not operated. During the first $0.5 \mathrm{~s}$ the knee is extending (lifting the body weight) while the other $0.5 \mathrm{~s}$ the knee is flexing (lowering the body weight). Therefore, during knee extension the MRF damper works by braking the leg inertia, while during knee flexion, the MRF damper works by braking the whole structure weight. At a first glance, the wave-form of the electrical power consumed by the SEA with and without the aid of the MRF brake is very similar to the mechanical power shown in Fig. 19. It can also be observed during the extension of the knee that the maximum instantaneous power consumed by the SEA without damper is similar as the one found in Fig. 19 (90 W). However, the maximum instantaneous power consumed by the SEA when the MRF damper helps braking the leg inertia is larger than the one obtained from mechanical power computation $(60 \mathrm{~W})$. The maximum energy saving $(30 \mathrm{~W})$ occurs at the end of the extension motion. Considering that the MRF damper consumed $11 \mathrm{~W}$ during its maximum braking torque, the overall maximum instantaneous energy saved is $19 \mathrm{~W}$ at the end of the extension motion (21\% of the maximum power used). Nevertheless, during the knee flexion the SEA employs a maximum of $23 \mathrm{~W}$ of electrical power to brake the knee, while using the MRF damper, the SEA consumes 


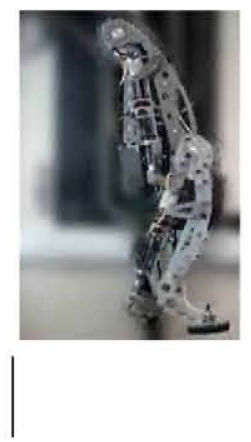

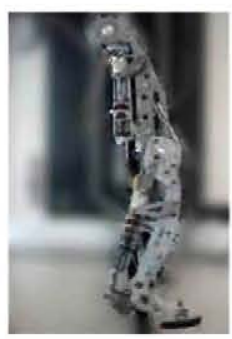

Stance
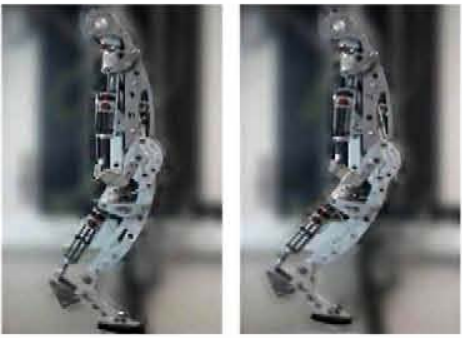

Toe off
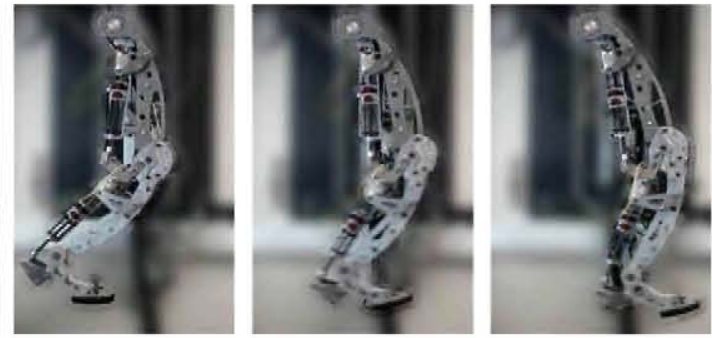

Swing

Fig. 21. Sequence of the locomotion cycle of the HADE leg.
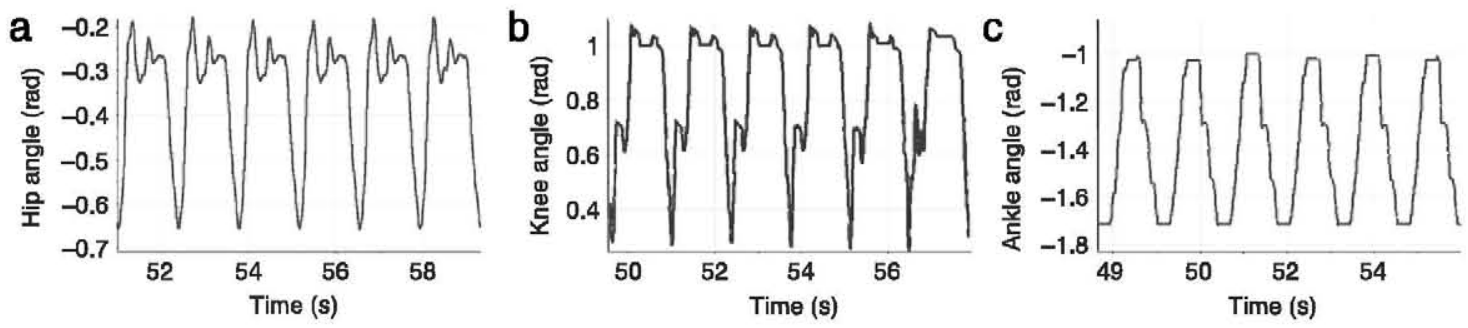

Fig. 22. Experimental joint trajectories of (a) hip; (b) knee; (c) ankle.

$16 \mathrm{~W}$ of electrical power. As the MRF brake consumes $11 \mathrm{~W}$, the power consumed by the MRF damper during knee flexion exceeds the energy savings at the SEA provided by the semi-active damping.

The average power consumed by the SEA in a support cycle without MRF damper is $25 \mathrm{~W}$, while the average power consumed by the SEA with the aid of the MRF damper is $17 \mathrm{~W}$. As the MRF damper consumes $11 \mathrm{~W}$ at its maximum activation state, then the total consumption of SEA and MRF damper is $28 \mathrm{~W}$, thus exceeding the power consumed by the SEA alone. However, we can state that, for the knee extension motion of the support phase, the use of MRF damper reduces the power consumption. The knee should then be damped passively during knee extension and left without passive brake during knee flexion for an efficient activation scheme. This scheme would be also improved by augmenting the maximum resistive torque of the MRF damper by means of gearing. This would multiply the resistive torque while maintaining the same power consumption at the MRF brake.

The last experiment was aimed at showing the natural-looking motion of the leg achieved with the herein proposed architecture. For this purpose, the performance of the locomotion cycle of the HADE leg was analyzed while tracking real trajectories. The leg was commanded to move using the locomotion controller and joint controller presented in this paper. Fig. 21 shows snapshots of the locomotion cycle. The video can be found in [46]. Fig. 22 shows joint trajectories during six locomotion cycles. In this experiment the leg is walking at a cycle speed of $0.54 \mathrm{~m} / \mathrm{s}$. Please note that the aim of this experiment is to measure the ability of the control architecture to follow reference trajectories in a smooth, natural fashion; it is not the goal of this experiment to test the maximum speed capabilities of the leg prototype. During this experiment the leg performed successfully following the reference commands in a natural-looking fashion, as it was one of the objectives of the gait controller proposed and the actuator selection.

The reported experimental results validate the performance of the proposed approach of combining SEAs and MR dampers for the efficient locomotion control of agile robot legs.

\section{Conclusions}

The success of the new generation of legged robots greatly depends on the development of strong and lightweight structures and specific actuators featuring large power-to-weight ratio, large torque-to-weight ratio and inherent compliance. Besides, a research effort on energy efficient locomotion controllers is required for the operation of these robots in the real world.

In this paper, the effectiveness of a combined actuation system based on series elasticity and MR dampers has been analyzed to achieve compliant, natural-looking motion in an energy efficient fashion. This combination of SEA and MR damping has been tested in a real leg prototype, the HADE leg. This leg has been designed as a leg for agile locomotion resembling the leg of a horse. In order to improve energy efficiency in the implementation of locomotion, the HADE project has proposed a control architecture whose main characteristic is the use of inherent robot dynamics for robot motion. In the inner control of actuators, direct force control and active compliance control strategies have been performed in order to provide compliant joint motion, which improve structural robustness and provide compliant interaction with the environment. Experimental results have shown that the use of an inner force loop in the active compliance controller provides a faster response, reduced settling time and a reduction of the steady state error compared to inner position loop impedance controllers, which are key factors for an efficient interaction control scheme. Experiments controlling the motion of the HADE leg assess the proposed design and control architecture. Besides, the reported experiments show minimal mechanical power used during the swing phase following the proposed control strategy. The combination of series elasticity and magneto-rheological damping allows for a $20 \%$ reduction of the power employed in braking the knee during knee extension motion of the support phase. However, the relatively large power consumption of the MRF device led us to suggest a combined damping mechanism during the leg support phase, where the MRF damper is activated during knee extension while released inactive during knee flexion. 
Here, it is more convenient to use SEA active damping instead for the sake of energy saving. These results report an improvement in efficiency for locomotion.

\section{Acknowledgments}

This work has been partially funded by the Spanish Ministry for Innovation and Science through grant DPI2010-18702 and by AECID through grant PCI-iberoamerica D/030531/10.

\section{References}

[1] T.E. Bihari, T.M. Wallister, M.R Patterson, Controlling the adaptive suspension vehicle, IEEE Computer 22 (6) (1989) 59-65.

[2] P. Gonzalez de Santos, E. Garcia, J. Estremera, Ouadrupedal Locomotion: An Introduction to the Control of Four-Legged Robots, Springer, London, 2006.

[3] Thornhill, et al. Design of an agile unmanned combat vehicle - a product of the DARPA UGCV program, in: G.R. Gerhart, C.M. Shoemaker, D.W. Gage (Eds.) Unmanned Ground Vehicle Technology V, in: Proceedings of SPIE, vol. 5083 2003, pp. 258-270.

[4] $\mathrm{K}$. Waldron, ]. Nichol, Architectural issues in running machines, in: ROMANSY, Montreal, Canada, 15, 2004, June 14-17.

[5] R.M. Alexander, A.S. Jayes, A dynamic similarity hypothesis for the gaits of quadrupedal mammals, Journal of Zoology 201 (1) (1983) 135-152.

[6] T.M. Griffin, R. Kram, S.J. Wickler, D.F. Hoyt, Biomechanical and energetic determinants of the walk-trot transition in horses, Journal of Experimental Biology 207 (24) (2004) 4215-4223.

[7] G. Carbone, M. Ceccarelli, Legged robotic systems, in: Cutting Edge Robotics, ARS Scientific Book, Wien, 2005, pp. 553-576.

[8] I. Poulakakis, J.A. Smith, M. Buehler, Modeling and experiments of untethered quadrupedal running with a bounding gait: the Scout II robot, International, Joumal of Robotics Research 24 (4) (2005) 239-256.

[9] P. Gonzalez de Santos, J. Galvez, J. Estremera, E. Garcia, SILO4-A true walking robot for the comparative study of walking machine techniques, IEEE Robotics and Automation Magazine 10 (4) (2003) 23-32. http://www.iai.csic.es/users/ silo4/.

[10] R. Hodoshima, T. Doi, Y. Fukuda, S. Hirose, T. Okamoto, J. Mori, Development of a quadruped walking robot TITAN XI for steep slope operation-Step over gait to avoid concrete frames on steep slopes, Journal of Robotics and Mechatronics 19 (1) (2007) 13-26.

[11] H. Kimura, Y. Fukuoka, A. Cohen, Adaptive dynamic walking of a quadruped robot on natural ground based on biological concepts, International Joumal of Robotics Research 26 (5) (2007) 475-490.

[12] L. Greenemeier, DARPA pushes machine learning with legged LittleDog robot Scientific American April 15, 2008

[13] Y. Fukuoka, H. Katabuchi, H. Kimura, Dynamic locomotion of quadrupeds Tekken3\&4" using simple navigation system, Joumal of Robotics and Mechatronics 22 (1) (2010) 36-42

[14] S.P.N. Singh, K.J. Waldron, Towards highfidelity onboard attitude estimation for legged locomotion via a hybrid range and inertial approach, in: B. Siciliano O. Khatib, F. Groen (Eds.), Springer Tracts in Advanced Robotics: Experimaental Robots IX, Springer, Germany, 2006, pp. 589-598.

[15] Z.G. Zhang, H. Kimura, Rush: a simple and autonomous quadruped running robot, Journal of Systems and Control Engineering 223 (Part I)(2009) 323-336.

[16] M. Raibert, K. Blankespoor, G. Nelson, R. Playter, BigDog, the rough-terrain quadruped robot, in: Proceedings of the 17th World Congress IFAC, Seoul, South Korea, 2008

[17] L Jackel, D. Hackett, E Krotkov, M. Perschbacher, J. Pippine, C. Sullivan, How DARPA structures its robotics programs to improve locomotion and navigation, Communications of the ACM 50 (11) (2007) 55-59.

[18] J. Estremera, K. Waldron, Thrust control, stabilization and energetics of quadruped running robot, International Joumal of Robotics Research 27 (10) (2008) 1135-1151.

[19] J. Hurst, J. Chestnutt, A. Rizzi, The actuator with mechanically adjustable series compliance, IEEE Transactions on Robotics 26 (4) (2010) 597-606.

[20] S.A. Migliore, L.H. Ting. S.P. Deweerth, Passive joint stiffness in the hip and knee increases the energy efficiency of leg swinging, Autonomous Robots 29 (2010) 119-135.

[21] J.W. Hurst, A. Rizzi, Series compliance for an efficient running gait, IEEE Robotics \& Automation Magazine (2008) 42-51.

[22] M. Focchi, E. Guglielmino, C. Semini, T. Boaventura, Y. Yang, D.G. Caldwell Control of a hydraulically-actuated quadruped robot leg, in: Proceedings of the IEEE International Conference on Robotics and Automation, Anchorage, AK USA, 2010.

[23] N. Nicholson, Gaits in general for dressage: math and variations on a theme of walk, trot, canter, in: Biomechanical Riding and Dressage: A Rider's Atlas, Zip Publishing, 2006.

[24] G. Hiebert, P. Whelan, A. Prochakzka, K. Pearson, Contribution of hind-limb flexor muscle afferents to the timing of phase transitions in the cat step cycle Journal of Neurophysiology 75 (1999) 1126-1137.

25] M. Hildebrand, Symmetrical gaits of horses, Science 150 (1965) 701-708.

26] J. Pratt, Exploiting inherent robustness and natural dynamics in the contro of bipedal walking robots, Ph.D. Thesis, Computer Science Department Massachusetts Institute of Technology, Cambridge, Massachusetts, USA, 2000

[27] R.V. Ham, T.G. Sugar, B. Vanderborght, K.W. Hollander, D. Lefeber, Compliant actuator designs, IEEE Robotics and Automation Magazine (2009) 81-94.
[28] M. Laffranchi, N. Tsagarakis, F. Cannella, D. Caldwell, Antagonistic and series elastic actuators: a comparative analysis on the energy consumption, in: Proceedings of the IEEE International Conference on Robotics and Automation, St. Louis, MO, USA, 2009, pp. 5678-5684.

[29] O. Eiberger, S. Haddadin, M. Weis, A. Albu-Schäffer, G. Hirzinger, On joint design with intrinsic variable compliance: derivation of the dlr qa-joint, in: Proceedings of the IEEE International Conference on Robotics and Automation, Anchorage, AK, USA, 2010, pp. 1687-1694.

[30] E. Dunn, R. Howe, Foot placement and velocity control in smooth bipedal walking, in: Proceedings of the IEEE International Conference on Robotics and Automation, Minneapolis, MN, USA, 1996, pp. 578-583.

[31] S. Kajita, F. Kanehiro, K. Kaneko, K. Yokoi, H. Hirukawa, The 3d linear inverted pendulum mode: A simple modeling for a biped walking pattern generation, in: Proc. Int. Conf. on Intelligent Robots and Systems, IROS, 2001, pp. 239-246.

[32] J. Pratt, C. Chew, A. Torres, P. Dilworth, G. Pratt, Virtual model control: an intuitive approach for bipedal locomotion, International journal of Robotics Research 20 (2) (2001) 129-143.

[33] R.N. Alexander, in: R.N. Alexander, G. Goldspink (Eds.), Terrestrial locomotion, Mechanics and Energetics of Animal Locomotion, Chapman and Hall, London, 1977.

[34] E. Garcia, J.C. Arevalo, F. Sanchez, J.F. Sarria, P. Gonzalez-de-Santos, Design and development of a biomimetic leg using hybrid actuators, in: IEEE/RS International Conference on Intelligent Robots and Systems, September 25-30, 2011, San Francisco, California.

[35] M. Hildebrand, The mechanics of horse legs, American Scientist 75 (6) (1987) 594-601.

[36] J. Pratt, B. Krupp, C. Morse, Series elastic actuators for high fidelity force control, Industrial Robot: An International Journal 29 (3) (2002) 234-241.

[37] D.W. Robinson, Design and analysis of series elasticity in closed-loop actuator force control, Ph.D. Thesis, Massachusetts Institute of Technology, Cambridge, Massachusetts, USA, 2000.

[38] E. Garcia, H. Montes, P. Gonzalez de Santos, Emerging actuators for agile locomotion, in: Proceedings of the International Conference on Climbing and Walking Robots, Istanbul, Turkey, 2009.

[39] D. Carlson, D. Catanzarite, K. St Clair, Commercial magneto-rheological fluid devices, in: Proceedings of ACTUATOR 2004, Bremen, Germany, 2004.

[40] N. Hogan, ]mpedance control: an approach to manipulation: Parts 1-3, ASME Journal of Dynamic Systems, Measurements and Control 107 (1985) 1-24

[41] B. Siciliano, L. Villani, Robot Force Control, Kluwer Academic Publishers, 1999.

42] T.A. McMahon, Muscles, Reflexes, and Locomotion, Princeton, New Jersey, USA 1984.

[43] J. Galvez, Perception, control and force distribution in legged robots, Ph.D. Thesis, Polytechnic University of Madrid, Spain, 2003.

[44] R.M. Alexander, Elastic Mechanisms in Animal Movement, Cambridge University Press, UK, 1988.

[45] S. Collins, A. Ruina, R. Tedrake, M. Wisse, Efficient bipedal robots based on passive-dynamic walkers, Science 307 (2005) 1082-1085.

[46] E. Garcia, The HADE project, 2007. http://www.iai.csic.es/users/egarcia/hade. html.

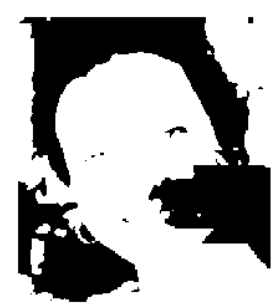

E. Garcia is a researcher at the Spanish National Research Council (CSIC) She received the B.E and Ph.D. degrees from the Universidad Politecnica de Madrid in 1996 and 2002, respectively. She joined the Department of Automatic Control of the Institute of Industrial Automation-CSIC in 1997 and she currently carries out her research activity in the Centre for Automation and Robotics (CSIC-UPM) since 2010. She has been a visiting scholar at the MIT Leg Laboratory in 1998 and at the Laboratoire d'Automatique de Grenoble in 2001. Her research is focused on improving the performance of legged robots, including dynamic stability of legged robots, active compliance in the foot-ground interaction, new actuators for legged robots, agile quadrupeds and lower-limb exoskeletons. She has participated in various research projects in legged locomotion like ROWER, a walking platform for ship building, SIL 04, a four legged locomotion system used as a testbed in most of her work, and DYLEMA, a project focused on a six legged walking platform for landmine detection and location. Currently, she is leading research with the direction of the HADE project on new actuation systems for achieving high-speed locomotion in walking robots, and ATLAS project for the development of the technology required to control lower-limb exoskeletons. Dr. Garcia is the co-author of a scientific book on quadrupedal locomotion, published by Springer in 2006 .

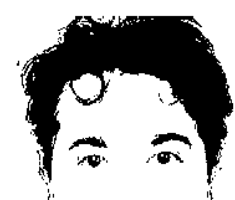

J.C. Arevalo is a Master Student in the Universidad Politecrica de Madrid. He received the B.E degree from the Universidad Simon Bolivar in 2010. In addition, he has been an exchange student in the Royal Institute of Technology (KTH) in Stockholm in (2008-2009), and a visiting scholar at the IHMC in 2009. Currently he carries out research activities in the Centre for Automation and Robotics (CSIC-UPM), where he is working in the HADE project on new actuation systems for achieving highspeed locomotion in walking robots, and ATLAS project for the development of the technology required to control lower-limb exoskeletons. He is focusing his research in improving human-machine interaction. 


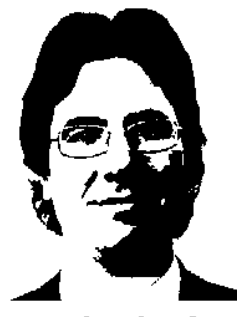

G. Mufioz is a Ph.D. Student at the Spanish National Research Council (CSIC). He received the Bachelor degree and Master degree from the Polytechnic University of Madrid in 2006 and 2008, respectively. He joined the Automatics, Electronics and Computer Science Department of the Polytechnic University of Madrid in 2002, working in four different projects; one funded by the University, two by the Spanish Ministry for Science and Technology (ROBINT and ROBONAUTA) and the third one by Airbus Spain, S.L. (CRAWLER). He has worked during these years in characterization of EMG signals for Parkinson disease, cognitive systems based on fuzzy logic for interactive robots and a full project from scratch to create a complete autonomous robot for non-destructive tests for aerospace industry. In 2011, he joined the Department of Automatic Control of the Centre for Automation and Robotics (CSIC) as a Ph.D. student. His current research is focused on improving the performance of agile quadrupeds based on the SLIP model. Currently, he is working in the HADE project on new actuation systems for achieving high-speed locomotion in walking robots, and ATLAS project for the development of the technology required to control lower-limb exoskeletons.

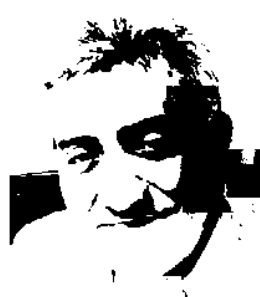

P. Gonzalez-de-Santos is a research professor at the Spanish National Research Council (CSIC). He received his Ph.D. degree from the University of Valladolid, Spain in 1986. Since 1981, he has been involved actively in the design and development of industrial robots and also in special robotic systems. His work during the past fifteen years has been focused on walking machines. He worked on the AMBLER project as a visiting scientist at the Robotics Institute of Carnegie Mellon University. Since then, he has been leading the development of several walking robots such as the RIMHO robot designed for intervention on hazardous environments, the ROWER walking machine developed for welding tasks in ship erection processes and the SIL 44 walking rohot intended for educational and basic research purposes. He has also participated in the development of other legged robots such as the REST climbing robot and the TRACMINER. Dr Gonzalez-de-Santos is now leading the DYLEMA project, that includes the construction of the SILO6 walking robot, to study how to apply walking machines to the field of humanitarian demining. 